\title{
Effect of TAT-signaling fusion system along with co-expression of GroEL/ES chaperones on secretory expression of somatropin
}

\author{
Mohammad Rabbani *, Razieh Ghasemi, Mohammad Reza Bagherinejad, Ali Jahanian \\ School of Pharmacy, Isfahan University of Medical Sciences, Isfahan, Iran
}

\begin{abstract}
Human growth hormone (somatropin) is one of the most widely used recombinant proteins that stimulates growth, cell reproduction, and cell regulation in humans. Synthetic production of this protein normally results in low yields and inclusion body formation. To overcome these difficulties, the production of somatropin along with two common signal peptides, namely TorA and SufI, in co-expression with a cytosolic chaperone, GroEL/ES, was evaluated in the present study. The target protein and the two signal sequences (TorA and SufI) were synthesized and cloned into an expression plasmid (pET-22) by using $N d e l$ and Xhol endonucleases. The expression vector (pGro7) containing chaperone proteins (GroES/EL) and one of the expression vectors containing the signal sequence (and the target protein) were co-expressed in the BL21 DE3 expression host. The results showed that although some of the expressed proteins exit the cytoplasm and enter the periplasmic space, there is also an accumulation of proteins (probably as inclusion body) inside the cytoplasmic area. Western blot analysis showed that the inclusion of a signal sequence in the cassette containing the target protein could help to secrete the protein in the periplasmic space and culture media when compared with control groups. The result of these experiments show that the TAT secretion system promotes transportation of the target protein out of the cytoplasm. This secretory system completes folding of the protein structure and transfers the mature protein to the periplasmic space.
\end{abstract}

Key words: chaperone, GroEL/ES, human growth hormone, somatropin, TAT system

\section{Introduction}

Escherichia coli is one of the most popular organisms for the expression and production of recombinant proteins. Because of numerous molecular tools and protocols such as a vast catalog of expression plasmids, a large number of engineered strains, and many cultivation strategies, $E$. coli is an organism of choice for the production and manipulation of recombinant proteins (Rosano and Ceccarelli, 2014). Despite its extensive use as a cell factory, the lack of eukaryotic posttranslational modifications, mis folding, and inclusion body formation are the main disadvantages of $E$. coli (Rosano and Ceccarelli, 2014). When conventional methods are used, proteins are often unrecoverable because of the formation of inclusion bodies or proteolytic degradation (Baneyx and Mujacic, 2004). Normally, inactive aggregate forms of the proteins are accumulated in the cytoplasm and more solubilizing/refolding steps are applied during the downstream process to obtain the correctly folded and active molecules. Therefore, engineering of transporting and folding pathways could address these limitations to produce more biologically active forms of proteins.

Human growth hormone ( $\mathrm{hGH}$ ) or somatropin is a single chain polypeptide containing 191 amino acid residues; it has a molecular mass of $22 \mathrm{kD}$ and is synthetized and secreted by the anterior pituitary gland. The endogenous growth hormone $(\mathrm{GH})$ does not need posttranslational modifications such as glycosylation, and therefore, E. coli host is considered as a prokaryotic choice for the industrial production of the recombinant hGH (rhGH) (Baneyx and Mujacic, 2004). The major concerns, however, with the use of $E$. coli as a host for the production of $\mathrm{hGH}$ are inclusion body formation, low recovery, and high cost of purification (Kim et al., 2013;

${ }^{*}$ Corresponding author: School of Pharmacy, Isfahan University of Medical Sciences, Isfahan, Iran; e-mail: rabanim@yahoo.com 
Levarski et al., 2014; Nguyen et al., 2014). To overcome the issues related to protein mis folding and inclusion body formation, several strategies have been proposed among which the co-expression of molecular chaperones facilitates correct folding of proteins and gene fusions in which fusion partners such as signal sequences facilitate transport of the proteins to the periplasm and culture media (de Marco et al., 2007).

It is well known that molecular chaperones and other folding catalysts facilitate protein folding and maintain protein homeostasis in vivo (Horwich, 2013). Molecular chaperones use ingenious mechanisms to prevent aggregation and promote efficient folding. The most abundant and functionally important classes of chaperones existing in E. coli are DnaK, DnaJ, GrpE, GroEL, and GroES (Grall et al., 2009). The GroEL/ES chaperonin system of $E$. coli has been studied extensively (Hartl and HayerHartl, 2009; Horwich, 2013). GroEL interacts with at least 250 different cytosolic proteins. Most of these proteins are in the size of 20 to $50 \mathrm{kDa}$ and have a complex $\alpha / \beta$ or $\alpha+\beta$ domain topologies (Fujiwara et al., 2010; Kerner et al., 2005). During overproduction of recombinant proteins, native chaperones may become scarce, whichresultsin inclusion body formation. However, if chaperones are co-expressed with recombinant proteins, they can help the preferred folding of recombinant proteins (Schlieker et al., 2002). Numerous studies on the co-expression of molecular chaperones with the recombinant proteins in $E$. coli have shown large quantities of correctly folded proteins (Goyal and Chaudhuri, 2015). Despite the large number of publications reporting the chaperone-dependentcontribution to increase the quantities of soluble target proteins in E. coli, there are no studies on attempts to fold $\mathrm{hGH}$ recombinant proteins that are overexpressed simultaneously with the help of coexpressed GroEL/ES chaperone inside E. coli cells. It has been shown that the co-expression of multiple chaperone systems results in increased solubility of some proteins up to 7-fold (de Marco et al., 2007).

In addition to the use of chaperones, signal sequences could also be used to help the secretion of proteins to the extracellular space; this could enable to minimize the degradation of proteins inside the cell. Fusion of the $\mathrm{N}$-terminus of the proteins with appropriate signal sequences could facilitate transportation of the proteins to the oxidative environment ofthe periplasm and culture media (Choi and Lee, 2004). By targeting the proteins to the outside of the cytoplasm, the downstream process in the industrial production of therapeutic proteins could be simplified and become more affordable and cost-efficient. The most common translocation systems in E. coli are the signal recognition particle (SRP)-dependent pathway and the TAT system (Klatt and Konthur, 2012; Silverman et al., 2012). The TAT pathway has a unique feature in transferring correctly fully/semi-folded proteins outside the cells. The SufI protein and trimethylamine $\mathrm{N}$-oxide reductase (TorA) are the two best-characterized prototype proteins exported by the $E$. coli TAT system (Bagherinejad et al., 2018).

Because there is no general rule for selecting a suitable signal peptide to ensure successful protein secretion, in the present study, we fused the N-terminus of hGH with one of the two signal peptides (TorA or SufI) and co-expressed the protein with the GroES/EL molecular chaperonin system.

Our main aim wasto improve the production of functional recombinant proteins by minimizing the formation of inclusion bodies by transporting the majority of proteins out of the cytoplasmic space.

\section{Materials and methods}

\section{Materials}

All chemicals and reagents were obtained from Merck (Darmstadt, Germany) and were of analytical grade. The culture media (Nutrient and Luria agar/broth) were purchased from Oxoid (Hampshire, UK), and chloramphenicol and ampicillin were obtained from Sigma Chemical Co. (USA). DNA polymerase (Taq and PFU polymerase) and endonucleases were purchased from Fermenras (Thermo Scientific, USA). Qiagen gel extraction kit was used for all purification from agarose gels according to the manufacturer's instructions. The primers were synthesized and purified by Tag Copenhagen A/S (Denmark).

\section{Strains and plasmids}

The constructs were synthesized by Biomatik (Canada) using E. coli optimization platform coding. E. coli strains were purchased from Invitrogen (USA). The expression vector $\mathrm{pET}$-22(b) was obtained from Novagen (Madison, USA). The chaperone plasmid set was obtained from TaKaRa (Japan). The pGro7 plasmid containing GroEL and GroES chaperones under the araB promoter was used in this study. 


\section{Cloning}

The hGH DNA sequence was obtained from www. drugbank.ca. After codon optimization, the sequences were ordered for chemical synthesis in fusion with the selected signal sequences (TorA and SufI). The pGEM plasmid encoding these sequences was constructed, and unique restriction enzymes $\mathrm{NdeI}$ and $\mathrm{XhoI}$ were introduced at the $5^{\prime}$ and $3^{\prime}$ ends, respectively. After plasmid preparation, pGEM plasmids were digested by $\mathrm{XhoI}$ and NdeI endonucleases. In parallel, plasmid pET-22(b) was digested by the same enzymes, and the cohesive ends of the vector fragments were re-ligated using $N d e \mathrm{I}$ and $\mathrm{XhoI}$ endonuclease, thus yielding either pET-22(b)-TorA-hGH or pET-22(b)-Sufl-hGH. His-tag was introduced at the C-terminus of somatropin to enhance purification and western blotting. The constructs were checked and confirmed by polymerase chain reaction (PCR) and digestion.

\section{Transformation}

Following plasmid preparation, an electroporation platform (GenePulser II, BioRad, USA) was used for co-transformation of the recombinant pET-22(b) and pGro7 plasmid (encoding chaperone) to the expression host strain $E$. coli BL21 (DE3). The electroporation system was set at $25 \mu \mathrm{F}, 2.5 \mathrm{kV}$, and $200 \Omega$. The pulse duration was $4.8 \mathrm{~ms}$. Following recoveryof the cells, selective plates containing ampicillin $20 \mu \mathrm{g} / \mathrm{ml}$ and chloramphenicol $34 \mu \mathrm{g} / \mathrm{ml}$ were spotted and streaked out with the tube content and incubated at $37^{\circ} \mathrm{C}$ for $24 \mathrm{~h}$. The transformed cells were transferred to a culture medium containing appropriate antibiotics (ampicillin for the pET-22(b) system and chloramphenicol for the TaKaRa system). To grow the resistant colonies, they were cultured overnight at $37^{\circ} \mathrm{C}$.

\section{Growth conditions}

A single colony from the streaked plate was inoculated in $10 \mathrm{ml}$ fluid LB medium containing appropriate antibiotics and incubated at $37^{\circ} \mathrm{C}$ and $200 \mathrm{rpm}$ overnight. This culture was used at $0.6 \mathrm{OD}$ as an inoculum for $50 \mathrm{ml}$ media containing ampicillin and chloramphenicol as the selective marker. Following induction by IPTG and L-arabinose at the final concentrations of $1 \mathrm{mM}$ and $4 \mathrm{mg} / \mathrm{ml}$, respectively, the cultures were re-incubated at $37^{\circ} \mathrm{C}$ and $200 \mathrm{rpm}$ for $4 \mathrm{~h}$. As control, the same procedure was applied for the cassette containing somatropin without any signal sequence and chaperone.

\section{Expression study}

Samples from the cytoplasm, periplasm, and culture media were prepared after 4 hours of induction to investigate the effects of signal sequence and chaperone on the translocation of hGH.

\section{Samples of culture media}

To study the secretion of somatropin in the culture medium, $1 \mathrm{ml}$ of the culture medium was centrifuged for 15 minutes at $7000 \mathrm{~g}$ and $4^{\circ} \mathrm{C}$. The supernatant was used for determining the secretory expression of somatropin into culture media.

\section{Periplasmic samples}

The pellet obtained from the centrifugation of the above media was used to prepare the periplasmic sample. Cold osmotic shock method was used for the extraction of the periplasmic content of the pellet to make the cells more penetrable, so that proteins could exit from the periplasmic space without damaging the cell membranes. For this purpose, $1 \mathrm{ml}$ of hypertonic solution (sucrose 25\%, EDTA $5 \mathrm{mM}$, Tris-HCl $50 \mathrm{mM}$ ) was added to the sample. The mixture was shaken on ice for $60 \mathrm{~min}$, and the supernatant was then collected and kept at $-20^{\circ} \mathrm{C}$ for protein analysis. Subsequently, $1 \mathrm{ml}$ of hypotonic solution $\left(\mathrm{MgCl}_{2}, 5 \mathrm{mM}\right)$ was added to the pellet and re-suspended and shaken for $60 \mathrm{~min}$. The cell suspension was centrifuged again for $15 \mathrm{~min}$ at $7000 \mathrm{~g}$ and $4{ }^{\circ} \mathrm{C}$, and the supernatant was kept at $-20^{\circ} \mathrm{C}$. The supernatants from the two steps of centrifugation was pooled and used as the periplasmic sample.

\section{Cytoplasmic extracts}

The pellet collected by centrifugation from the previous step was re-suspended in phosphate buffer (100 mM, pH 7.2). The cells were sonicated (Hielscher, Germany) on ice for 5 times (10 s), 80\% amplitude with 10 s intervals. Following sonication, the suspension was centrifuged for 15 minutes at $7000 \mathrm{~g}$ and $4^{\circ} \mathrm{C}$, and the supernatant was collected and kept at $-20^{\circ} \mathrm{C}$ as the cytoplasmic fraction.

\section{Preparation of cell samples for protein product analyses by western blotting}

Proteins extracted from culture medium, periplasmic, and cytoplasmic fractions were subjected to sodium dodecyl sulphate-polyacrylamide gel electrophoresis (SDSPAGE) and concomitant western blotting, as described 
Table 1. Sequences of forward and reverse primers used in this study

\begin{tabular}{l|l|l}
\hline Reference & \multicolumn{1}{|c|}{ Description/Sequence $\left(5^{\prime} \rightarrow 3^{\prime}\right)$} & \multicolumn{1}{c}{ Primers } \\
\hline This study & CAT ATG AACAACAAC GAT CTGTTT CA & TorA-hGH Forward \\
\hline This study & CTC GAG TTA GTG GTGGTGGTG GT & TorA-hGH \& SufI-hGH Reverse \\
\hline This study & CAT ATG TCA CTG AGT CGT CGC CA & SufI-hGH Forward \\
\hline This study & CAT ATG TTT CCG ACG ATC CCG CTG & hGH Forward \\
\hline This study & CG CTC GAG TTA AAA GCC ACA ACT ACC TTC & hGH Reverse \\
\hline
\end{tabular}

elsewhere (Sambrook et al., 1989). SDS-PAGE was performed in 15\% (w/v) acrylamide-resolving gel with 5\% stacking. Proteins were stained by Coomassie brilliant blue R-250. Western blotting was performed using diaminobenzidine and anti-hGH monoclonal antibody (CinnaGen, Iran) to confirm the expression of hGH.

\section{Results}

\section{The structure of expression cassettes}

The pGEM plasmids harboring the signal sequences and hGH were first digested by $\mathrm{XhoI}$ and NdeI to ensure integrity of the ordered sequences. The insert in plasmids containing hGH without signal sequences was $599 \mathrm{bp}$ in length, whereas the lengths of Sufl-hGH and TorA-hGH were 650 and 700 bp, respectively. After confirmation of the target sizes, the requested sequences were amplified by PCR using the primers given in Table 1. For the PCR reaction, the optimum conditions were determined through the gradient method. Following digestion by $\mathrm{NdeI}$ and $\mathrm{XhoI}$, the inserted fragments were ligated to pET-22(b). The recombinant plasmids were co-transfectedto $E$. coli $\mathrm{BL} 21$ (DE3) with the pGro7 plasmid from the TaKaRa kit containing GroEL and GroES chaperones.

\section{Expression study}

Figure 2 presents the SDS-PAGE analysis of various fractions (cytoplasm, periplasm, and culture). As shown in Figure 2, when no signal sequence was used, the hGH protein was mostly retained in the cytoplasmic fraction (Lane II). The molecular weight of hGH is $22 \mathrm{kDa}$. When the signal sequences were used to co-express hGH with GroEL/ES chaperones, the amount of proteins secreted to different fractions was different from the above mentioned control. As shown in Figure 3 and Figure 4, when TorA and SufI were used as signal sequences, the target

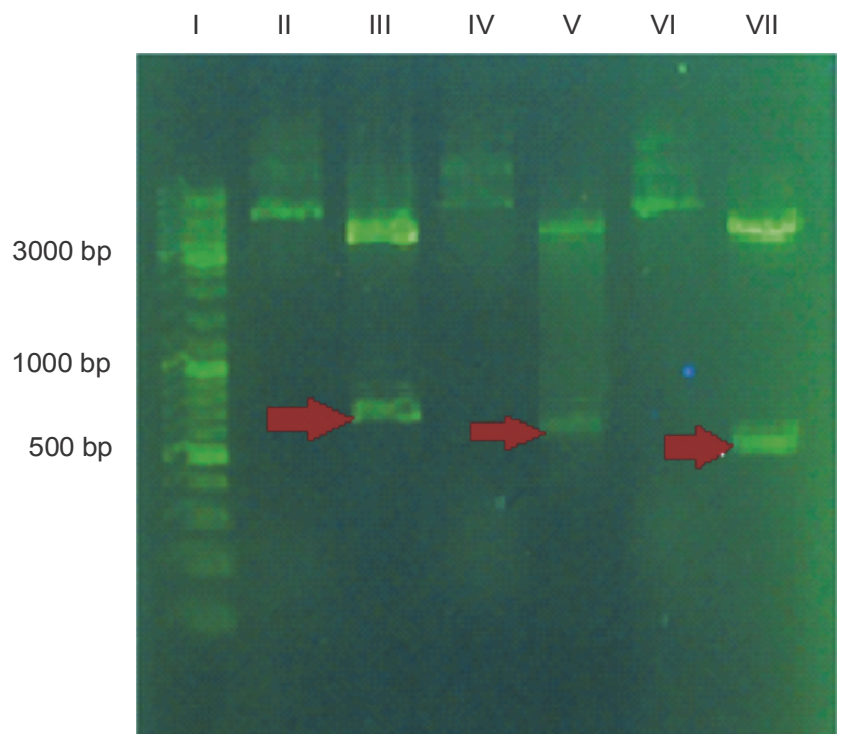

Fig. 1. NdeI and XhoI digestion profile of pGEM bearing hGH with or without signal sequences. The expected sizes of hGH, TorA-hGH, and Sufl-hGH are 599, 700, and 650 bp, respectively. Lanes: I - DNA ladder mix; II - uncut pGEM plasmid containing hGH-TorA; III - pGEM plasmid containing hGHTorA digested with NdeI and XhoI; IV - uncut pGEM plasmid containing hGH-SufI; V - pGEM plasmid containing hHG-SufI digested by XhoI and NdeI; VI - uncut pGEM plasmid containing hGH; VII - pGEM plasmid containing hGH digested with NdeI and XhoI

protein exited the cytoplasm and entered the periplasm. Although some proteins remained in the cytoplasmic space of a cell, the amount of proteins leaving the cell was much greater than the control expression when no signal sequences were used (Fig. 2). Certainly, these processes require optimization to achieve the maximum excretion of protein of interest. The use of hypertonic solution, for example, yielded better results than hypotonic solution with regard to the exit of the target protein to the cytoplasmic environment. 


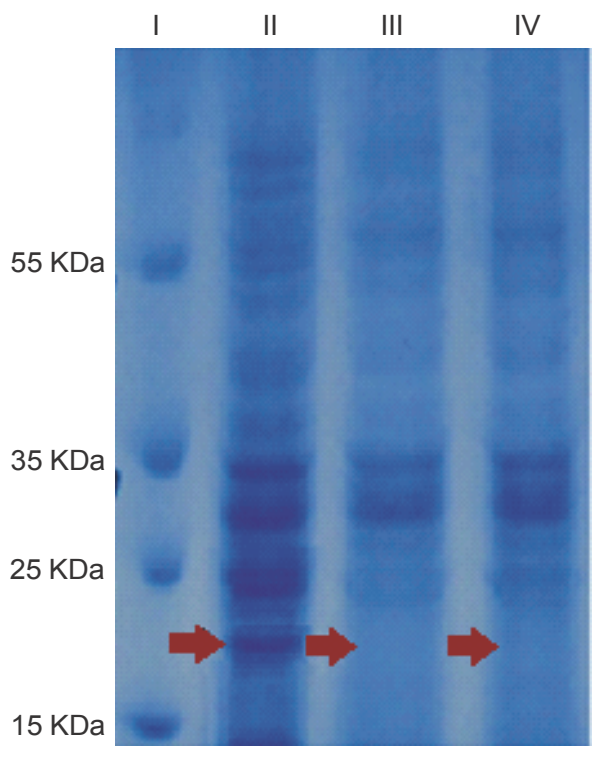

Fig. 2. SDS-PAGE analysis of the hGH samples obtained from different fractions. Expression pattern of hGH without signal sequences on polyacrylamide gel. Lanes: I - molecular weight marker; II - cytoplasmic sample; III - periplasmic sample; and IV - samples of culture fraction. The molecular weight of hGH is $22 \mathrm{kDa}$

Western blot analysis of TorA-hGH co-expressed with GroEL/ES chaperones is shown in Figure 5. Lane II and lane III show the periplasmic fraction of hGH following osmotic shock with hypertonic and hypotonic solutions, respectively. As shown in the figure, $\mathrm{hGH}$ was present neither in the periplasm nor in the culture fractions (lane IV). However, when hGH was analyzed in the cytoplasmic fraction, a sharp band of approximately $22 \mathrm{kDa}$ was observed (Fig. 5, lane V). In the presence of TorA as a signal sequence and GroEL/ES chaperones, the hGH pattern of expression differed from that of the afore mentioned control. In Figure 5, lane VI to lane IX illustrate the pattern of $\mathrm{hGH}$ expression in culture, periplasmic, and cytoplasmic fractions. Although the target protein wasexported to the periplasmic space and outside the cells, most of the proteins were localized in the cytoplasm. The hypertonic solution eluted more proteins than the hypotonic solution.

Western blot analysis of Sufl-hGH co-expressed with GroEL/ES chaperones is shown in Figure 6. In the presence of Suflas a signal sequence and GroEL/ES chaperones, the hGH pattern of expression differed from the control. In Figure 6, lane I to lane V illustrate the pattern of hGH expression in culture medium, periplasmic

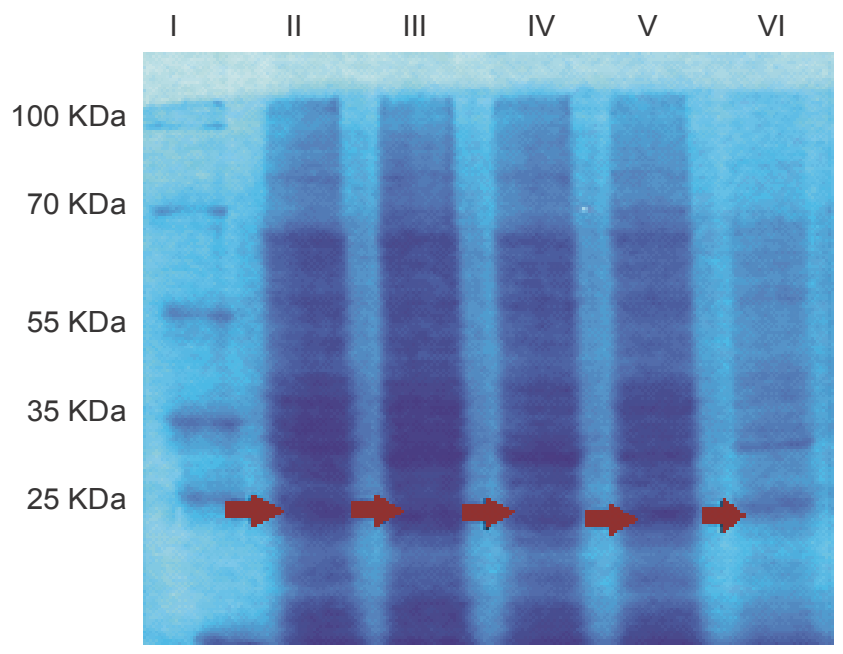

Fig. 3. Expression pattern of hGH when TorA wasused as a signal sequence. Co-expression of TorA-hGH and the GroEL/ES chaperone protein following osmotic shock in hypotonic and hypertonic solutions. Lanes: I - protein molecular weight marker; lanes II and III - are different samples from cytoplasmic fractions; IV - periplasmic fraction following osmotic shock with a hypotonic solution; $\mathrm{V}$ - periplasmic fraction following osmotic shock with a hypertonic solution; VI - secretory fraction to culture fraction

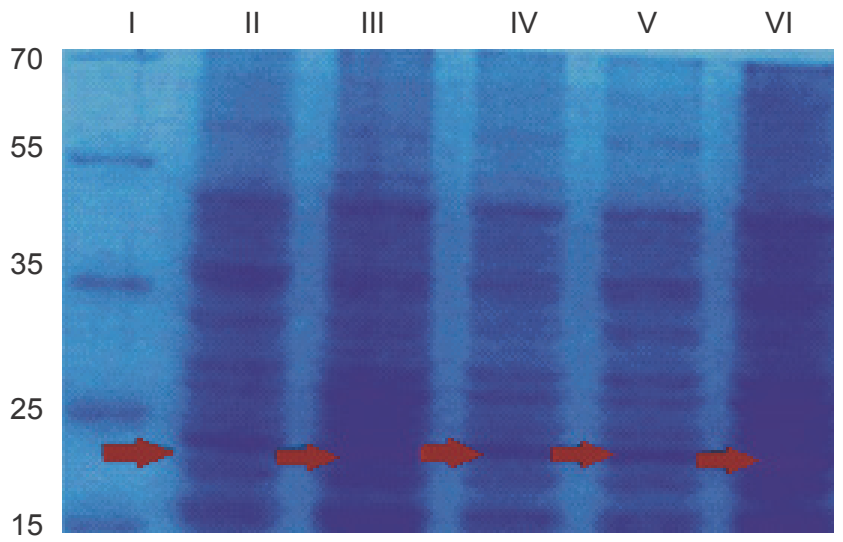

Fig. 4. Expression pattern of hGH when SufI wasused as a signal sequence. Co-expression of Sufl-hGH and the GroEL/ES chaperone protein following osmotic shock in hypotonic and hypertonic solutions. Lanes: I - protein molecular weight marker; II - periplasmic fraction following osmotic shock with a hypotonic solution; III - periplasmic fraction following osmotic shock with a hypertonic solution; lanes IV and V - are different samples from secretory fractions to culture fraction; VI - cytoplasmic fraction

cytoplasm and fractions. Although many of the target proteins were still located in the cytoplasm, the amount of secreted protein in hypertonic solution eluted was higher than in hypotonic solution. 


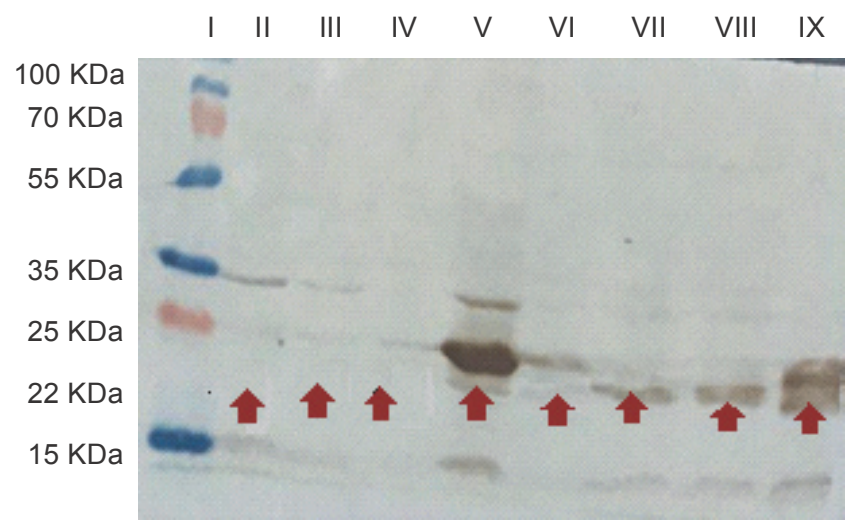

Fig. 5. Western blot analysis of TorA-hGH co-expressed with GroEL/ES chaperones. Co-expression of TorA-hGH and GroEL/ES chaperonesunder the conditions described in the Methods section. Lanes: I - protein molecular weight marker; II - periplasmic fraction following osmotic shock with a hypertonic solution of hGH; III - periplasmic fraction following osmotic shock with a hypotonic solution of hGH; IV - secretory fraction to culture fraction for $\mathrm{hGH} ; \mathrm{V}$ - cytoplasmic fraction for hGH; VI - secretory fraction to culture fraction of hGH when using TorA as a signal sequence; VII - periplasmic fraction following osmotic shock with a hypotonic solution of hGH when using TorA as a signal sequence; VIII - periplasmic fraction following osmotic shock with a hypertonic solution of hGH when using TorA as a signal sequence; IX - cytoplasmic

fraction of hGH when using TorA as a signal sequence

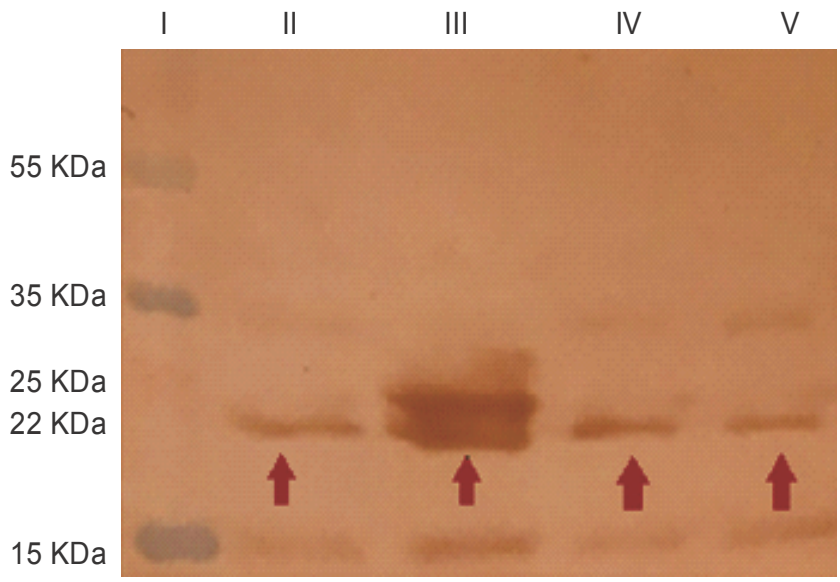

Fig. 6. Western blot analysis of Sufl-hGH co-expressed with GroEL/ES chaperones. Co-expression of Sufl-hGH and GroEL/ES chaperonesunder the conditions described in the Methods section. Lanes: I - protein molecular weight marker; II - secretory fraction to culture fraction of hGH when using SufI as a signal sequence; III - cytoplasmic fraction of hGH when using SufI as a signal sequence; IV - periplasmic fraction following osmotic shock with a hypertonic solution of hGH when using SufI as a signal sequence; V - periplasmic fraction following osmotic shock with a hypotonic of hGH when using SufI as a signal sequence
Thus, as shown in these figures, when the SUfI or TorA signal sequences were fused to the growth hormone for co-expression with GroEL/ES chaperones, significant amounts of the expressed proteins were released outside the cytoplasmic space.

\section{Discussion}

A major concern with the expression of recombinant proteins in E. coli is the formation of insoluble aggregates known as inclusion bodies. Additional steps such as solubilization and refolding are normally required to be added to the procedures prior to the purification schemes. Recovery of biologically active proteins from the aggregated state is a time-consuming and costly procedure. In such cases, maximizing the yields of therapeutic proteins in the periplasmic space or culture fraction could become an attractive method for overcoming the above concern. Different strategies have been used to transmit the recombinant protein to the periplasmic space or culture fraction. Fusion of the protein with a signaling peptide and co-expression with chaperone proteins, which help in correct folding of the proteins, is an attractive strategy. The present study aimed to investigate the effect of TorA and SufI signal sequences on the secretory expression of hGH. Moreover, because molecular chaperones are known to interact with folding intermediates and facilitate protein folding, we systematically investigated the effect of GroEL/ES chaperones to gain further insight on the process of inclusion body formation in E. coli.

In control cells transfected with plasmids with no signal sequences added to $\mathrm{hGH}$, most $\mathrm{hGH}$ proteins were accumulated as inclusion bodies in the cytoplasm. Co-overexpression of plasmid-encodingTorA-hGH or SufI-hGH and GroEL/ES chaperone led to the increase in the levels of the proteins in the periplasm and culture medium. Although not all proteins exited the cytoplasmic space under tested conditions, the secretion rate and recovery could be improved by optimizing the processes. Culture conditions such as temperature, $\mathrm{pH}$, and culture fraction compositions have enormous effects on the export of proteins out of the cells. Choi and Lee (2004) showed that reductionof the induction temperature could markedly solubilize most of the inclusion bodies of somatropin under optimized culture conditions in the periplasmic space (Choi and Lee, 2004). 
The TAT pathway has been used with very promising results for exporting the protein of interest to outside the cytoplasm (Matos et al., 2014). This strategy could offer several advantages, including a proper disulfide bond formation, more biologically active form of the protein, and improved product quality by lowering contamination with nucleic acids, lipopolysaccharides, and host cell proteins. Various recombinant proteins such as cMyc, KLF4, Oct4, Sox2, antibodies, GFP, alkaline phosphatase (PhoA), phytate containing 4 disulfide bonds $(\mathrm{AppA})$, anti-interleukin $1 \beta \mathrm{scFv}$, and growth hormone have used this system (TAT pathway) for secreting their target protein into the cytoplasm and periplasmic space (Kang et al., 2012; Low et al., 2013; Matos et al., 2014; Mergulhao et al., 2005; Stanley et al., 2000). The highly distinctive feature of this system is that correctly folded proteins could be exported to the periplasmic space. Thus, it is one kind of a quality control system that could actively differentiate correctly folded active form of the proteins from inactive ones.

It is not possible to evaluate the direct influence of GroEL/ES folding machine on the folding of the fusion protein in the current setting. Nevertheless, secretion of the protein to the periplasmic space and culture medium is only possible through the anti-aggregating activity of the chaperones, which is also evident in other studies (Bagherinejad et al., 2018). It is well established that GroEL/ES chaperone has disaggregation activity based on its ability to renature heat-treated RNA polymerase (Ziemienowicz et al., 1993). It has also been shown that the co-expression of GroEL/ES markedly enhances soluble and functional expression of recombinant human interferon-gamma in E. coli (Yan et al., 2012). Through the continuous assistance of the GroEL/ES chaperonin system, a large number of subunits have the opportunity to assemble the homodimer correctly. Expression studies using western blotting analysis and SDS-PAGE confirmed that somatropin could be expressed by both cassettes. However, the pattern was different for the two signal sequences. Western blotting results showed that the TorA signal sequence could transport more expressed proteins to the periplasmic space and culture fraction than the SufI signal sequence.

\section{Conclusions}

Overall, our results indicate that (I) fusing the heterologous protein with TAT signal sequences and co-expres- sion with chaperones could export the recombinant protein to outside of the cytoplasmic space and reduce inclusion body formation and (II) TorA signal sequence was more active in transporting the target protein to the culture fractionthan the SufI signal sequence. These data confirmed previous work regarding the potential of the TAT pathway for industrial applications. These findings suggest that the TAT secretion system promotes transportation of the target protein out of the cytoplasm. This secretory system completes folding of the protein structure, transfers the mature protein to the periplasmic space, and helps in its transformation.

\section{References}

Bagherinejad M.R., Sadeghi H.M.-M., Abedi D., Moazen F., Rabbani M. (2018) Effect of twine-arginine translocationsignaling fusion system and chaperones co-expression on secretory expression of somatropin. Adv. Biomed. Res. 7.

Baneyx F., Mujacic M. (2004) Recombinant protein folding and misfolding in Escherichia coli. Nat. Biotechnol. 22(11): 1399-1408.

Choi J., Lee S. (2004) Secretory and extracellular production of recombinant proteins using Escherichia coli. Appl. Microbiol. Biotechnol. 64(5): 625-635.

de Marco A., Deuerling E., Mogk A., Tomoyasu T., Bukau B. (2007) Chaperone-based procedure to increase yields of soluble recombinant proteins produced in E. coli. BMC Biotechnol. 7(1): 32.

Fujiwara K., Ishihama Y., Nakahigashi K., Soga T., Taguchi H. (2010) A systematic survey of in vivo obligate chaperonindependent substrates. EMBO J. 29(9): 1552-1564.

Goyal M., Chaudhuri T.K. (2015) GroEL-GroES assisted folding of multiple recombinant proteins simultaneously over-expressed in Escherichia coli. Int. J. Biochem. Cell Biol. 64: 277-286.

Grall N., Livny J., Waldor M., Barel M., Charbit A., Meibom K.L. (2009) Pivotal role of the francisella tularensis heatshock sigma factor rpoh. Microbiology 155(8): 2560-2572.

Hartl F.U., Hayer-Hartl M. (2009) Converging concepts of protein folding in vitro and in vivo. Nat. Struct. Mol. Biol. 16(6): 574-581.

Horwich A.L. (2013) Chaperonin-mediated protein folding. J. Biol. Chem. 288(33): 23622-23632.

Kang D.G., Kim C.S., Seo J.H., Kim I.G., Choi S.S., Ha J.H., Nam S.W., Lim G., Cha H.J. (2012) Coexpression of molecular chaperone enhances activity and export of organophosphorus hydrolase in Escherichia coli. Biotechnol. Prog. 28(4): 925-930.

Kerner M.J., Naylor D.J., Ishihama Y., Maier T., Chang H.C., Stines A.P., Georgopoulos C., Frishman D., Hayer-Hartl M., Mann M. et al. (2005) Proteome-wide analysis of chaperonin-dependent protein folding in Escherichia coli. Cell 122(2): 209-220. 
Kim M.J., Park H.S., Seo K.H., Yang H.J., Kim S.K., Choi J.H. (2013) Complete solubilization and purification of recombinant human growth hormone produced in Escherichia coli. PLoS One 8(2): e56168.

Klatt S., Konthur Z. (2012) Secretory signal peptide modification for optimized antibody-fragment expression-secretion in Leishmania tarentolae. Microb. Cell Fact. 11: 97.

Levarski Z., Soltysova A., Krahulec J., Stuchlik S., Turna J. (2014) High-level expression and purification of recombinant human growth hormone produced in soluble form in Escherichia coli. Protein Expr. Purif. 100: 40-47.

Low K.O., Mahadi N.M., Illias R.M. (2013) Optimisation of signal peptide for recombinant protein secretion in bacterial hosts. Appl. Microbiol. Biotechnol. 97(9): 3811-3826.

Matos C.F., Robinson C., Alanen H.I., Prus P., Uchida Y., Ruddock L.W., Freedman R.B., Keshavarz M.E. (2014) Efficient export of prefolded, disulfide bonded recombinant proteins to the periplasm by the tat pathway in Escherichia coli cydisco strains. Biotechnol. Prog. 30(2): 281-290.

Mergulhao F., Summers D.K., Monteiro G.A. (2005) Recombinant protein secretion in Escherichia coli. Biotechnol. Adv. 23(3): 177-202.

Nguyen M.T., Koo B.K., Thi Vu T.T., Song J.A., Chong S.H., Jeong B., Ryu H.B., Moh S.H., Choe H. (2014) Prokaryotic soluble overexpression and purification of bioactive human growth hormone by fusion to thioredoxin, maltose binding protein, and protein disulfide isomerase. PLoS One 9(3): e89038.
Rosano G.L., Ceccarelli E.A. (2014) Recombinant protein expression in escherichia coli: advances and challenges. Front Microbiol. 5: 172.

Sambrook J., Fritsch E.F., Maniatis T. (1989) Molecular cloning: a laboratory manual. Cold Spring Harbor Laboratory Press.

Schlieker C., Bukau B., Mogk A. (2002) Prevention and reversion of protein aggregation by molecular chaperones in the E. coli cytosol: implications for their applicability in biotechnology. J. Biotechnol. 96(1): 13-21.

Silverman J.M., Brunet Y.R., Cascales E., Mougous J.D. (2012) Structure and regulation of the type vi secretion system. Ann. Rev. Microbiol. 66: 453-472.

Stanley N.R., Palmer T., Berks B.C. (2000) The twin arginine consensus motif of tat signal peptides is involved in secindependent protein targeting in Escherichia coli. J. Biol. Chem. 275(16): 11591-11596.

Yan X., Hu S., Guan Y.-X., Yao S.-J. (2012) Coexpression of chaperonin GroEL/GroES markedly enhanced soluble and functional expression of recombinant human interferongamma in Escherichia coli. Appl. Microbiol. Biotechnol. 93(3): 1065-1074.

Ziemienowicz A., Skowyra D., Zeilstra-Ryalls J., Fayet O., Georgopoulos C., Zylicz M. (1993) Both the Escherichia coli chaperone systems, GroEL/GroES and DNAk/DNAj/ GRPE, can reactivate heat-treated RNA polymerase. Different mechanisms for the same activity. J. Biol. Chem. 268(34): 25425-25431. 